\title{
A Novel Hybrid Carbon Price Forecasting Model Based on Radial Basis Function Neural Network
}

\author{
S. WANG* J.W. E AND S.G. LI \\ College of Mathematics and Information Science, Shaanxi Normal University, Xi'an 710119, China \\ (Received April 5, 2018; in final form July 3, 2018)
}

\begin{abstract}
In the wake of the stronger and stronger development of carbon market, the carbon price fluctuation has drawn the attention of researchers, encouraging numerous researchers involved in the carbon price study. Owing to the strongly nonstationary and nonlinear characteristics of carbon price, most of existing approaches failed to forecast the carbon price perfectly. In our study, a novel hybrid forecasting model is presented to forecast the carbon price. Variational mode decomposition (VMD) and independent component analysis (ICA) are utilized to preprocess the chosen data for getting the independent components. Then the independent components are trained by radial basis function neural network (RBFNN) to predict them respectively. Finally, the forecasting result is obtained by linear combination. In addition, the numerical results show that the VMD-ICA-RBFNN model outperforms wavelet-based NN, VMD-RBFNN, EMD-ICA-RBFNN, RBFNN, ARIMA-GARCH and ARIMA models.
\end{abstract}

DOI: 10.12693/APhysPolA.135.368

PACS/topics: forecasting, carbon price, radial basis function neural network, variational mode decomposition, independent component analysis

\section{Introduction}

With the appearance of carbon emission reduction financial products, numerous investment agencies swarm into the carbon financial market, advancing the movement of the market. During the process of the evaluation of the market, carbon price is the center issue. It is necessary of presenting effective forecasting methods to anticipate the complex and irregular trend of the carbon trading market [1].

In the recent years, there exist several forecasting models in terms of temporal sequence. Autoregressive moving average (ARMA) model is an effective forecasting model, while it is a stationary model, making the forecasting exist several limitations. For this, the ARMA model has been generalized to the nonstationary autoregressive integrated moving average (ARIMA) model to deal with those limitations. Furthermore, the generalized autoregressive conditional heteroskedasticity (GARCH) prediction model has been introduced to forecast the time series, whose effect has also been measured [2]. However, the forecasting models above cannot capture the nonlinear characteristics of some time series, for example, carbon price series, while artificial neural network (ANN) [3-7] is more suitable to forecast the nonlinear time series thanks to the strong self-learning and adaptive capabilities, including RBFNN and BPNN, which have achieved the goals in forecasting successfully. What is more, RBFNN has better forecasting performance than BPNN due to its simpler network shapes, faster exercise routines and finer approaching abilities [6, 7]. Among the single prediction models above, RBFNN has distinct advantages in forecasting.

\footnotetext{
*corresponding author; e-mail: shuang_wang@snnu.edu.cn
}

Carbon price series are nonlinear, nonstationary and contain several affecting factors [8]. It is not sufficient for forecasting the carbon price series accurately using the single models above. So the combined forecasting models have been put forward, involving some methods of decomposition like empirical mode decomposition (EMD) [9] and multi-resolution wavelet analysis $[10,11]$. Using the two methods mentioned, the data can be processed for several sub-series with different scales $[9,12]$. The operation decreases the difficulties of grasping the characteristics of complex carbon price series. However, there exists the problem of frequent appearance of mode mixing in EMD and undesired spikes all over the frequency scales in wavelettransform. In 2014, a novel non-recursive mode decomposition method VMD was presented [13]. VMD conquers the drawback of EMD and shows prominent power of noise robustness and mode decomposition. ICA is a computational algorithm to obtain the independent inherent sources from time series as well as signals, which has been widely used in financial data analysis [14]. The integrated EMD-ICA model was proposed, showing the good performance of decomposing single-channel signals, which was applied in massive different areas [15]. Thus, based on given above, the EMD-ICA model can be improved to be the VMD-ICA model, which possesses the merits of VMD and ICA: strong signal separation and factors extraction power.

Owing to the remarkable performance of VMD-ICA model in mode decomposition and underlying factors extraction of nonlinear and nonstationary time series, as well as the accurate forecasting effect of RBFNN, this paper firstly presents a novel hybrid forecasting model: VMD-ICA-RBFNN model. 


\section{Methodology \\ 2.1. $V M D$}

VMD is an entirely non-recursive mode decomposition model, firstly introduced in 2014 [13]. In terms of VMD, the purpose we would like to achieve is to disintegrate one original valued series $x$ into several sub-series (modes) $u_{k}$. It is of great significance to evaluate the bandwidth of a mode. From this, the main work of VMD is the constrained variational problem

$$
\begin{aligned}
& \min _{\left\{u_{k}\right\},\left\{u_{k}\right\}} \sum_{k=1}^{K}\left\|\partial(t)\left[\left(\delta(t)+\frac{\mathrm{j}}{\pi t}\right) u_{k}(t)\right] \mathrm{e}^{-\mathrm{j} \omega_{k} t}\right\|_{2}^{2}, \\
& \text { s.t. } \sum_{k=1}^{K} u_{k}=x,
\end{aligned}
$$

here $\left\{u_{k}\right\}:=\left\{u_{1}, u_{2}, \cdots, u_{K}\right\}$ and $\left\{\omega_{k}\right\}:=$ $\left\{\omega_{1}, \omega_{2}, \cdots, \omega_{K}\right\}$ are the set of sub-signals and the centre frequencies of sub-signals respectively, $\delta(t)$ is the Dirac distribution, $K$ represents the amount of modes, $\mathrm{j}$ is imaginary unit and $\mathrm{j}^{2}=-1$.

\section{2. $I C A$}

ICA is a statistical technique for obtaining the inherent sources from the original signal. In 1994, Common [16] proposed the concept formally.

Assume the observed data samples $x_{i} \in R^{n}$ are generated via a linear mixture of source signals $s_{i} \in R^{n}$ with zero mean. In matrix notion, the basic model of ICA is as following [17]:

$$
X=A S=\sum_{i=1}^{K} a_{i} s_{i}^{T}
$$

where $X=\left[x_{1}, x_{2}, \cdots, x_{K}\right]^{T} ; A=\left[a_{1}, a_{2}, \cdots, a_{K}\right]^{T}$ is a mixing matrix, and $a_{i}$ is the $i$-th column of $A$, $i=1,2, \cdots, K ; S=\left[s_{1}, s_{2}, \cdots, s_{K}\right]^{T}$. In the model, we cannot have a knowledge of prior information of $A$ and $S$ in general. The structure diagram of the basic ICA model is presented as Fig. 1.

$$
\begin{aligned}
& S=\left(\begin{array}{c}
s_{1} \\
s_{2} \\
\vdots \\
s_{K}
\end{array}\right) \Rightarrow A \Rightarrow X=A S \Rightarrow B \Rightarrow Y=B X=\left(\begin{array}{c}
y_{1} \\
y_{2} \\
\vdots \\
y_{K}
\end{array}\right) \\
& \text { source mixture observed separation separated } \\
& \text { signal system signal system signal }
\end{aligned}
$$

Fig. 1. The structure diagram for ICA.

Hence the main purpose of ICA is to get an estimation of $A$, and then get an estimation of $A^{-1}$ written $B$ called separation matrix, such that the generated data $Y=B X=\left[y_{1}, y_{2}, \cdots, y_{K}\right]^{T}$ is the estimation of $S$. For solving the problem, we adopt the method of maximization of nongaussianity by measuring the independence of
ICs. Due to the complex computation of negentropy, the approximation of negentropy is introduced as follows [17]:

$$
J(y) \propto[E\{G(y)\}-E\{G(v)\}]^{2},
$$

where $y$ is a random variable such that $E\{y\}=0$ and $\operatorname{Var}\{y\}=1, v \sim N(0,1), G(y)=y \cdot \exp \left(-\frac{y^{2}}{2}\right)$. Benefitting from the efficient and accurate performance, we choose the algorithm of FastICA to obtain the estimated matrix $B$ [17].

\section{3. $R B F N N$}

RBFNN is a class of artificial neural networks. The learning of RBFNN is equal to finding a hook face to well fit training sample data in the hyperspace, while it can be generalized to the processing for using the multidimensional hook face to interpolate the data. Hence it can be regarded as the problem of curve-fitting or approximation in the hyperspace. Based on neural networks, radial basis function is a function set. The basic model of RBFNN is made up of input, hidden, and output layer, and the roles of them are entirely different [7]. The first layer, called input layer, is responsible for connecting the network with external part which consists of several units. The second layer is hidden layer, whose function is to do the processing of nonlinearity switch from the former layer to this layer, where radial basis functions (RBF) come into play. Most likely, this layer is high-dimensional. The third layer is linear and responds to the action modes acted on the input layer. The input layer is related to the output layer as

$\psi_{i}(x)=\sum_{i=1}^{M} \omega_{i} G\left(x, c_{i}\right)=\sum_{i=1}^{M} \omega_{i} \exp \left(\frac{-1}{\sigma_{i}^{2}}\left\|x-c_{i}\right\|^{2}\right)$,

where $M$ stands for hidden units' number, $x$ denotes the input vector, $\psi_{i}(x)$ represents the output of unit $i, G(\cdot)$ denotes the activation function, $\omega_{i}$ and $c_{i}$ represent the weight between hidden and output layer and the center vector of the $i$-th hidden layer, respectively. In Eq. (4), the Gaussian function is applied to act as the activation function, where $\sigma_{i}$ is the width of the $i$-th unit. The main goal of training RBFNN is to minimize the errors between the practical and target output as

$$
e=\frac{1}{2} \sum_{k=1}^{N}\left(t_{k}-y_{k}\right)^{2}
$$

here $y_{k}$ is the practical output, $t_{k}$ is the target output. The basically complete RBFNN forecasting process consists of four steps:

Firstly, input the sample data and fall the samples into training and testing samples.

Secondly, train the network using the training samples, and here, so as to select the structure of network and the hidden nodes centers, $k$-means clustering algorithm [7] is adopted.

Thirdly, test the forecasting accuracy of the trained network according to Eq. (5), if the precision is not enough, return to the second step.

Finally, output the forecasting results. The RBFNN forecasting flow chart is illustrated as Fig. 2. 


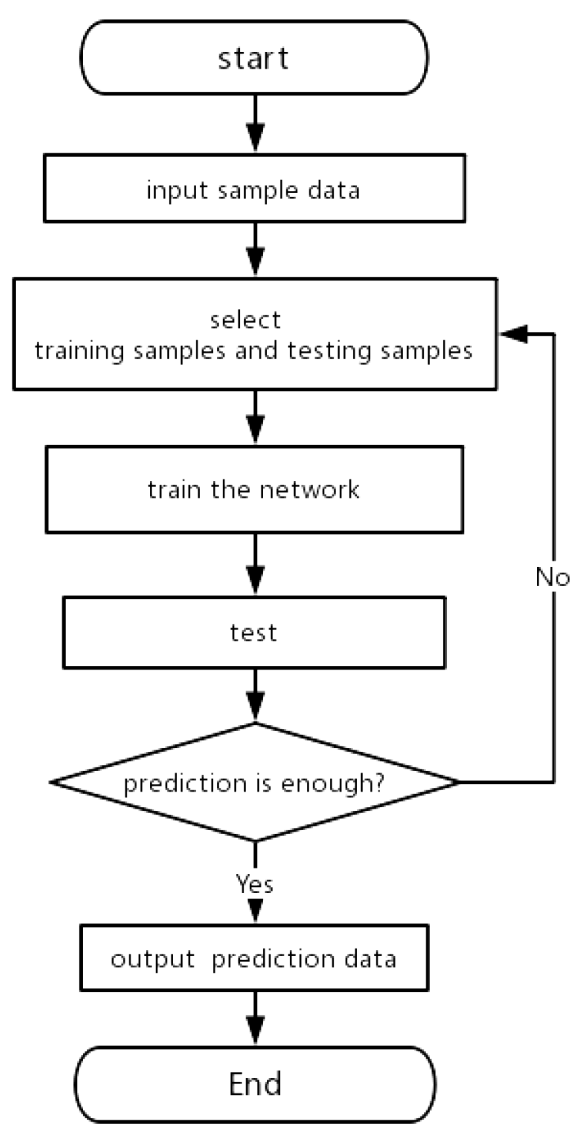

Fig. 2. RBFNN forecasting flow chart.

\section{The hybrid VMD-ICA-RBFNN}

In most forecasting methods, the models using neuron networks are significantly efficient. Reference [7] indicates that BPNN and RBFNN forecasting model are better than several linear models, while RBFNN performs more efficient than BPNN [4, 7]. Obviously, the RBFNN model has definite advantages in forecasting.

However, practical time series, for example, carbon price data, the series has strongly nonlinear and nonstationary characteristics [1]. The single forecasting models cannot achieve the goals perfectly. Thereupon, several integrated models were introduced with EMD, which is introduced to combine with the single forecasting models to improve the accuracy. But there exists a serious problem in EMD: the frequent appearance of boundary effect as well as mode mixing. So in our hybrid method, we introduce the technique of VMD, avoiding the disadvantages in EMD, with better effect [13].

After the process of VMD, ICA is utilized in our hybrid method. As a vital method for finding the inherent factors from the original signals, ICA has been given great attention by researchers and applied in multifarious areas [14]. In 2010, EMD and ICA were combined to propose the EMD-ICA model for single-channel signals processing, showing good decomposition performance [15], while the components extracted are independent mutually. The model assists various statistic analysis. To avoid the drawback in EMD, the EMD-ICA model can be substituted to be the VMD-ICA model, which possesses the merits of VMD and ICA. Owing to the remarkable performance of VMD-ICA model in mode decomposition and underlying factors extraction for some nonlinear and nonstationary time series, as well as the accurate forecasting effect of RBFNN, this paper presents a novel hybrid forecasting model: VMD-ICA-RBFNN model.

Additionally, a reconstruction algorithm using the transformative relative hamming distance (RHD) [18] is applied to IMFs. We reconstruct the modes IMFs decomposed from VMD by evaluating the contribution coefficient $C C I_{i}$ of the $i$-th IMF, $i=1,2, \cdots, K$, as Eq. (6), the goal of which is to measure the importance of the $i$-th IMF on the effect of reconstruction.

$$
C C I_{i}=1-\frac{1}{P-1} \sum_{t=1}^{P-1} Q(t),
$$

where $Q(t)=1$ if $(f(t+1)-f(t))(\hat{f}(t)-f(t)) \geq 0$, or else $Q(t)=0 ; \hat{f}(t)=\sum_{i \neq k} v_{i}(t) ; f(t)$ stands for the original carbon price data; $v_{i}(t), i=1,2, \cdots, K$ refer to IMFs; according to the theory of $\mathrm{VMD}, \sum_{i=1}^{K} v_{i}(t)=f(t)$. Moreover, we set a hard threshold $\lambda$. The contribution coefficient of the $i$-th IMF $C C I_{i}$ is compared with $\lambda$. We sum the IMFs whose $C C I_{i}$ is less than $\lambda$ while other IMFs remain unchanged. In general, the threshold $\lambda$ is determined to be a small value like $0.2,0.3[18]$ and in this study we make it 0.3 . In this way, a new set of series is obtained. The main steps of the VMD-ICA-RBFNN model are as following:

Step 1: Decompose the carbon price data $f(t)$ by VMD to get $K$ IMFs, $v_{i}(t), i=1,2, \cdots, K$;

Step 2: Reconstruct the IMFs to get one novel set of series according to $C C I_{i}, i=1,2, \cdots, K$, after that, extract the ICs, $s_{k}(t), k=1,2, \cdots, M, M \leq K$, via ICA;

Step 3: Apply the RBFNN model to every independent component (IC), with the output $r_{k}(t), k=$ $1,2, \cdots, M$. The output is the forecasting results of every IC;

Step 4: Reconstruct the outputs of the previous step for having the forecasting results of the original carbon price data by the linear combination as following:

$$
y(t)=\sum_{k=1}^{M} b_{k} r_{k}(t),
$$

here $y(t)$ is the final prediction result of $f(t), b_{k}$ is the sum of the $k$-th column of $A$. In fact, by the process of VMD, reconstruction algorithm and ICA, the estimated IMFs and ICs are obtained, while we get the estimated original data $\tilde{f}(t)$ as Eq. (8).

$$
\tilde{f}(t)=\sum_{i=1}^{K} v_{i}(t)=\sum_{k=1}^{M} b_{k} s_{k}(t) .
$$

Equation (7) is derived from Eq. (8).

Step 5: Evaluate the forecasting results by the evaluation criteria introduced in Sect. 4.2. 


\section{Empirical analysis}

\subsection{Data and evaluation criteria}

In this paper, the authors introduce the EUA price matured in December, 2017 (DEC17) in the Intercontinental Exchange (ICE) (www.theice.com). Here we use the carbon price series DEC17 from 26 November 2013 to 18 July 2017, excluding public holidays, with 939 sample data. In the proposed hybrid forecasting model, the former 681 sample data and the latter 258 sample data are used to be the training sets and the testing sets, respectively, to certify the accuracy of the forecasting model via the evaluation criteria. The DEC17 we choose is demonstrated as Fig. 3, indicating the strong nonlinearity and nonstationarity of carbon price data.

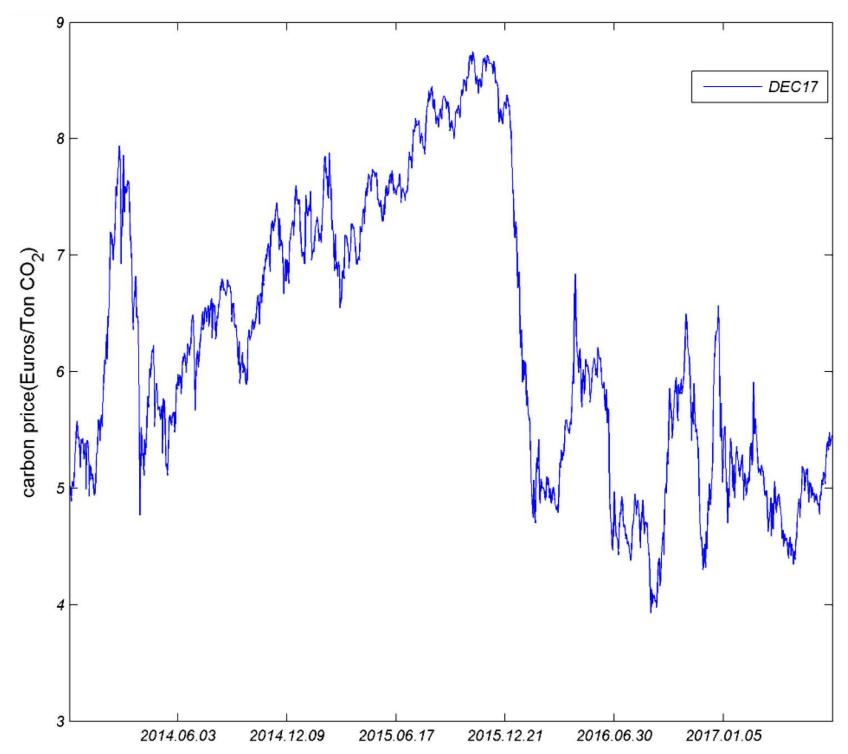

Fig. 3. Dec17 from 26 November 2013 to 18 July 2017.

\subsection{Evaluation criteria}

For measuring the deviation of forecasting, three evaluation indexes that have been chosen in [19] are introduced: mean absolute error (MAE), root mean square error (RMSE) and maximum absolute percentage error (MAPE).

At the same time, we adopt the Diebold-Mariano (DM) test [20] for the purpose of drawing a comparison about forecasting situation among diverse models. The authors have a knowledge of the DM statistic like

$$
\mathrm{DM}=\frac{\bar{d}}{\sqrt{V_{\bar{d}} / N}},
$$

where $\bar{d}=\frac{1}{N} \sum_{t=1}^{N}\left[g\left(e_{1}(t)\right)-g\left(e_{2}(t)\right)\right] ; \quad g\left(e_{i}(t)\right)=$ $\sum_{t=1}^{N} e_{i}(t)^{2}, e_{i}(t)=x(t)-x_{i}(t), i=1,2 ; V_{\bar{d}}$ is an estimation as to $\bar{d} ; x_{1}(t)$ and $x_{2}(t)$ show the series by testing model and other one model, respectively.

\subsection{Forecasting results}

The forecasting experiments are conducted by the technique of MATLAB R2014a. In terms of VMD, the parameters are as following (the name of the parameters are consistent with the original design in VMD MATLAB procedure): alpha is 4000 ; tol is $10^{-7}$; tau is 0.3 ; init is 2 . But it is a difficult task to ensure the value of modes $K$, because there exists no theoretical foundation in VMD model [13]. As is discovered in [4], too large number of modes may make the processing program burdened and long while too small that may result in that the modes decomposed by VMD cannot represent the characters of the original carbon price data. By repeated experiments, we determine $K$ is 10 , the setting of which makes the forecasting results wonderfully accurate and other parameters are reasonable. As for ICA, we choose the FastICA algorithm. Moreover in the procedure of RBFNN, the number of hidden layer is determined by the Matlab library function: newrb function. In terms of every IC, the relevant parameters are not uniform completely.

As is illustrated in Sect. 3, we take five steps to perform the forecasting experiments.

First, VMD is applied to decompose the carbon price sample data DEC17 into ten IMFs as Fig. 4.

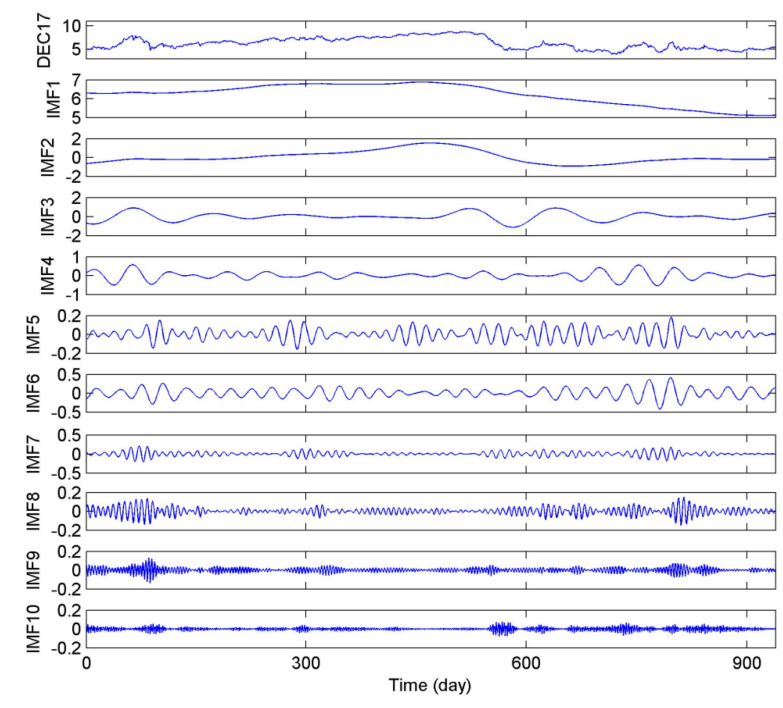

Fig. 4. The IMFs of DEC17 decomposed by VMD.

TABLE I

The contribution coefficients of the IMFs for the DEC 17.

\begin{tabular}{c|c|c|c|c}
\hline \hline$C C I_{1}$ & $C C I_{2}$ & $C C I_{3}$ & $C C I_{4}$ & $C C I_{5}$ \\
\hline 0.4919 & 0.3786 & 0.3522 & 0.2692 & 0.1497 \\
\hline \hline$C C I_{6}$ & $C C I_{7}$ & $C C I_{8}$ & $C C I_{9}$ & $C C I_{10}$ \\
\hline 0.1327 & 0.1016 & 0.0846 & 0.0714 & 0.0799
\end{tabular}

Then, IMF4 to IMF10 are combined while a new series is obtained, according to the $C C I_{i}, i=1,2, \cdots, K$ in Table I. After that, we extract ICs via the method of ICA as Fig. 5. 

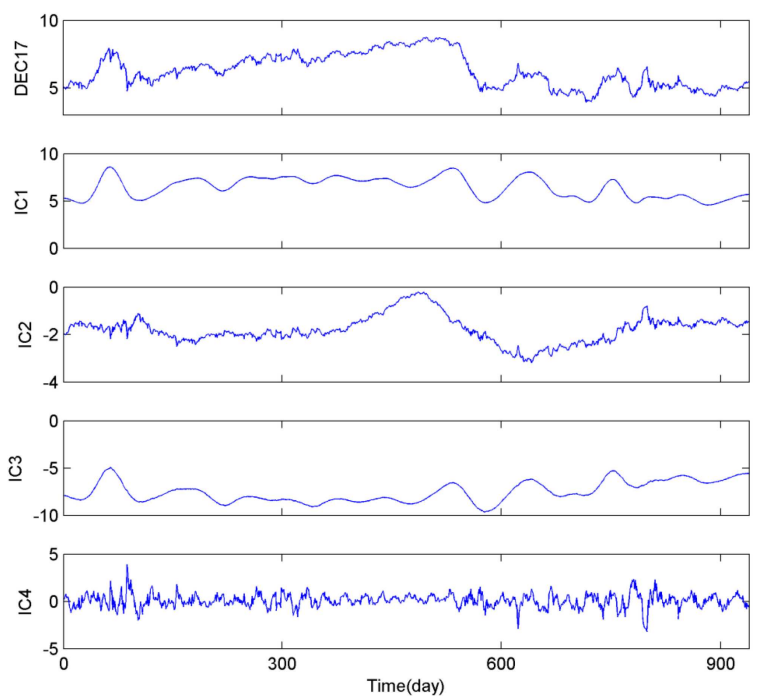

Fig. 5. The ICs of DEC17 disposed by ICA.

At the same time, the authors have a knowledge of the evaluated series of DEC17 after that process by means of VMD-ICA. We can use the calculation for MAE, RMSE, MAPE to show the perfect evaluation for original series via VMD-ICA technique. MAE is 0.0209 , RMSE is $0.0330, \mathrm{MAPE}$ is 0.0031 . The results above tell that it is not necessary of taking notice of the errors resulting from the VMD-ICA operation and the final forecasting results in the end show that the advantages of VMD-ICA can cover the little errors.

Third, apply the RBFNN forecasting model to every IC to get the forecasting result $r_{k}(t), k=1,2,3,4$ as Fig. 6 .
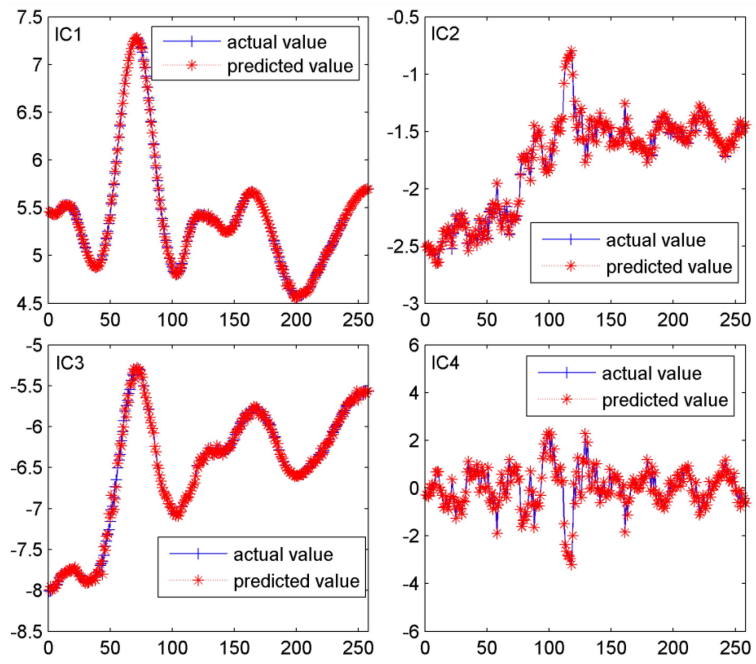

Fig. 6. RBFNN forecasting results of ICs.

Fourth, reconstruct the forecasting results as Eq. (7), where according to the results of ICA we get the mixing matrix $\mathrm{A}$ as Eq. (10).

$$
A=\left(\begin{array}{cccc}
-0.1810 & -0.0554 & -0.4493 & 0.4256 \\
-0.9180 & -0.1710 & -0.2096 & 0.0388 \\
0.0135 & 0.0149 & -0.2711 & -0.2433 \\
-0.0550 & 0.2967 & -0.0228 & -0.0009
\end{array}\right)
$$

From this, taking the mixing matrix $A$ to Eq. (7), the final forecasting result can be gained as

$$
\begin{gathered}
y(t)=\sum_{k=1}^{4} b_{k} r_{k}(t)=-1.1405 r_{1}(t)+0.0852 r_{2}(t) \\
-0.9527 r_{3}(t)+0.2211 r_{4}(t) .
\end{gathered}
$$

The predictive effect is illustrated in Fig. 7 .

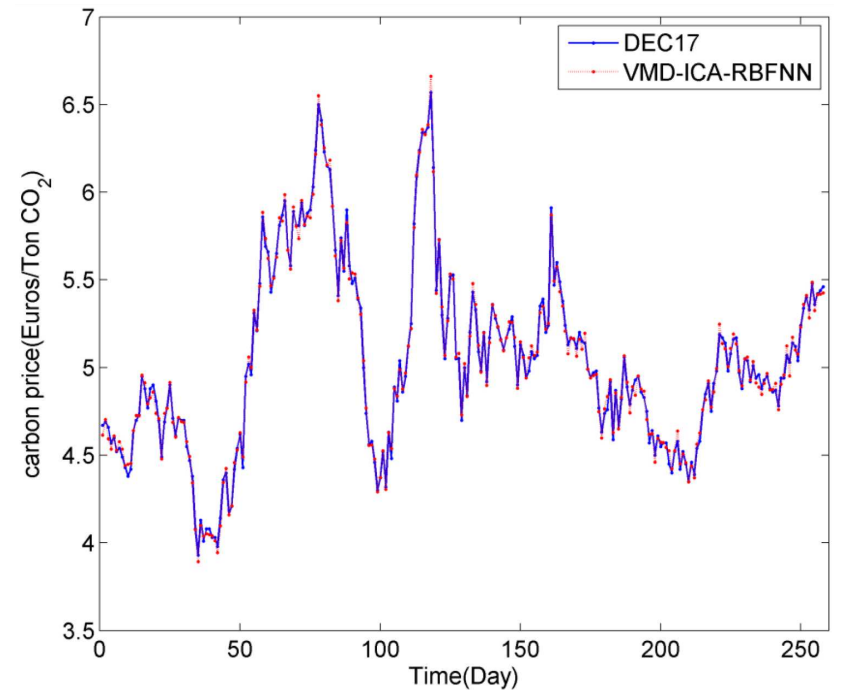

Fig. 7. VMD-ICA-RBFNN prediction effect picture.

Finally, we do the operation of evaluating the final prediction result of DEC17 by the proposed hybrid method. Here, due to showing the good predicting effect, we employ six types of forecasting models, ARIMA, ARIMAGARCH, RBFNN, wavelet-based neural networks (WNN), VMD-RBFNN and EMD-ICA-RBFNN models, to compare with VMD-ICA-RBFNN model. Firstly, we conduct the ARIMA model adopting the statistical software package of Eviews and adopt the ARIMA (2, 1, $3)$ model. Secondly, after the examination of residuals of the ARIMA $(2,1,3)$ model, there exists the characteristic of autoregressive conditional heteroskedasticity. Hence, based on the ARIMA $(2,1,3)$ model, the authors establish the GARCH $(1,1)$ model. After the operation of the $\operatorname{GARCH}(1,1)$, the result of the variance equation is obtained as Eq. (12):

$$
\begin{array}{cccc}
\sigma_{t}^{2}= & 3.345 \times 10^{-6}+ & 0.1169 \mu_{t-1}^{2}+ & 0.8988 \sigma_{t-1}^{2} \\
\text { Z-test } & 2.329 & 10.475 & 113.056
\end{array}
$$

Thirdly, RBFNN model has been mentioned above, which can be used for forecasting directly. The fourth one, with respect to the VMD-RBFNN model, we conduct the RBFNN to every mode decomposed in the experiment of VMD-ICA-RBFNN, and reconstruct the results of the forecasting by summing the forecasting result of every mode. 
Fifthly, in the model of the W-NN model, first, the prediction experiment is performed by the multi-resolution wavelet analysis and the decomposition result is shown as Fig. 8. Next, the hurst exponent [11] of each of component is measured as Table II.
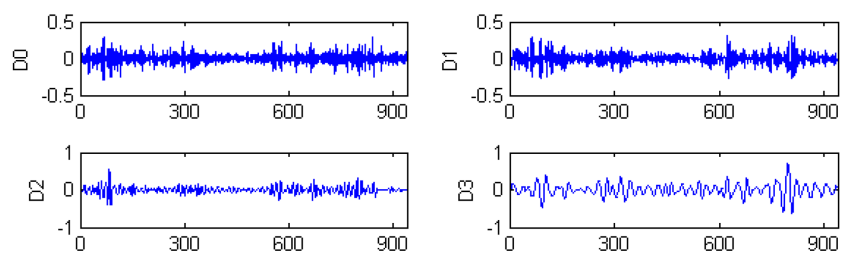

I $\int_{-1}^{1} \underset{300}{600}$


Fig. 8. The decomposition results of DEC17 by the wavelet transform.

According to the results in Table II, the components: D0, D1, and D2, whose hurst exponents are close to 0 , are removed, on account of the poor significance for the predictability. At last, the remaining components are trained by the RBFNN, respectively, and the results are added. Sixthly, the method of applying the EMDICA-RBFNN model to DEC17 is similar to the proposed VMD-ICA-RBFNN, where we use the technique of EMD to replace VMD and the parameters for EMD are ensured as $[\theta, \theta, \alpha]=[0.05,0.5,0.005]$. The comparison results are shown in Table III and Table IV.

From the results in Table III, the three evaluation indexes of our proposed hybrid forecasting model VMDICA-RBFNN model are all the least, thus it is obvious that VMD-ICA-RBFNN model is the most accurate in the forecasting methods considered. In detail, the authors conclude: (1) The forecasting performance WNN, VMD-RBFNN, EMD-ICA-RBFNN and VMD-ICARBFNN models are all better than the two single model
ARIMA, RBFNN and the combined model ARIMAGARCH, indicating the mode decomposition and independent features extraction technique meaningful in the process of forecasting the complex time series like carbon price data. (2) The ARIMA and ARIMA-GARCH method are worse than the RBFNN model. In fact, $\mathrm{Yu}$ et al. pointed that the ARIMA model is not proper to predict the heavily nonlinear time series as a type of linear model in 2010 [19]. Even though the technique of GARCH fixes the errors in the ARIMA model to some extent, the performance is worse than RBFNN, verifying the strong nonlinear function approximation capability. (3) The VMD-RBFNN model is better than RBFNN model, which indicates variational mode decomposition strategy improves the forecasting performance. (4) The prediction performance of VMD-ICA-RBFNN model is more accurate than the W-NN, VMD-RBFNN and EMDICA-RBFNN models. The results demonstrate that the VMD algorithm outperforms the EMD algorithm in decomposition and ICA method is conducive to advance the accuracy and precision of forecasting by independent features extraction.

TABLE II

The hurst exponents of the components obtained by the wavelet transform.

\begin{tabular}{c|c|c|c|c}
\hline \hline D0 & D1 & D2 & D3 & D4 \\
\hline-0.1875 & -0.1856 & 0.0120 & 0.2732 & 0.3989 \\
\hline \hline D5 & D6 & D7 & D8 & A0 \\
\hline 0.6292 & 0.7175 & 0.8779 & 0.9935 & 0.9915
\end{tabular}

TABLE III

Evaluation indexes comparison of disparate models.

\begin{tabular}{l|c|c|c}
\hline \hline $\begin{array}{c}\text { Forecasting } \\
\text { models }\end{array}$ & MAE & MAPE & RMSE \\
\hline ARIMA & 0.1350 & 0.0267 & 0.1780 \\
ARIMA-GARCH & 0.1040 & 0.0169 & 0.1656 \\
RBFNN & 0.0733 & 0.0148 & 0.1374 \\
W-NN & 0.0702 & 0.0122 & 0.1269 \\
VMD-RBFNN & 0.0536 & 0.0106 & 0.0775 \\
EMD-ICA-RBFNN & 0.0413 & 0.0080 & 0.0556 \\
VMD-ICA-RBFNN & 0.0277 & 0.0055 & 0.0374
\end{tabular}

DM test results of disparate proposed models.

TABLE IV

\begin{tabular}{l|c|c|c|c|c|c}
\hline \hline \multicolumn{1}{c|}{$\begin{array}{c}\text { Testing } \\
\text { models }\end{array}$} & ARIMA & ARIMA-GARCH & RBFNN & W-NN & VMD-RBFNN & EMD-ICA-RBFNN \\
\hline ARIMA-GARCH & 0.0000 & & & & \\
RBFNN & 0.0000 & 0.0000 & & & \\
W-NN & 0.0000 & 0.0000 & 0.0115 & & & \\
VMD-RBFNN & 0.0000 & 0.0000 & 0.0000 & 0.0000 & & 0.0000 \\
EMD-ICA-RBFNN & 0.0000 & 0.0000 & 0.0000 & 0.0000 & 0.0000 & 0.0000 \\
VMD-ICA-RBFNN & 0.0000 & 0.0000 & 0.0000 & 0.0000 & \\
\hline
\end{tabular}

Note: The $p$-values of the DM-test are shown in this table. When the $p$-value is smaller than 0.05 , the test rejects the original hypothesis, certifying the fact that there is a significant difference between the compared forecasting models. 
According to the results in Table IV, we can find the obvious difference in the side of prediction performance, which suggest the momentous meaning of our included three basic methods in our hybrid model. In total, the combination of the three basic parts VMD, ICA, and RBFNN is of great significance in carbon price forecasting.

\section{Conclusions}

In our study, a novel VMD-ICA-RBFNN multiscale forecasting model is presented to predict the carbon price. The process of pretreatment VMD-ICA contributes to decreasing the difficulties of forecasting. RBFNN is proposed to do the work of predicting. For highlighting the accuracy of the proposed model, ARIMA, ARIMA-GARCH, RBFNN, WNN, VMD-RBFNN and EMD-ICA-RBFNN are compared with it. Based on the experiments, the VMD-ICARBFNN model shows the best performance of forecasting. The fact that the pretreatment process of VMDICA improves the accuracy of forecasting and decreases the difficulties from the nonlinearity and nonstationarity of complex carbon price series is well shown. Accordingly, the proposed forecasting model VMD-ICARBFNN model is valuable for predicting the carbon price series.

\section{Acknowledgments}

The authors are grateful to the anonymous reviewers and editors. This work is supported by the National Natural Science Foundation of China (Grant No. 11771263).

\section{References}

[1] IEA, Appl. Phys. Lett. 109, 1275 (2016).

[2] Y. Wang, C. Wu, Energy Econom. 34, 2167 (2012).
[3] B. Zhang, C. Richard, J. Marwan Anwar, D. Dominik, F. Barry M, IEEE Trans. Neural Networks 12, 765 (2001).

[4] S. Lahmiri, J. Computat. Sci. 12, 23 (2015).

[5] X. Zheng, T. Chen, Commun. Theor. Phys. 40, 165 (2003).

[6] A.H. Isik, N. Isik, Acta Phys. Pol. A 129, 516 (2016).

[7] D.K. Ranaweera, N.F. Hubele, A.D. Papalexopoulos, IEE Proc. Gen. Transmiss. Distribut. 142, 45 (1995).

[8] Y.J. Zhang, Y.M. Wei, Appl. Energy 87, 1804 (2010).

[9] N.E. Huang, Z. Shen, S.R. Long, M.C. Wu, H.H. Shih, Q. Zheng, N.C. Yen, C.T. Chi, H.H. Liu, Proc. Math. Phys. Eng. Sci. 454, 903 (1998).

[10] K.K. Minu, M.C. Lineesh, C. Jessy John, Appl. Math. Sci. 4, 2485 (2010).

[11] D. Boubacar, A. Kodjo, C. Alben, Renew. Energy 92, 202 (2016).

[12] A. Tealab, H. Hefny, A. Badr, Future Comput. Inform. J. 2, 39 (2017).

[13] K. Dragomiretskiy, D. Zosso, IEEE Trans. Signal Process. 62, 531 (2014).

[14] A. Hyvarinen, E. Oja, Neural Networks 13, 411 (2000).

[15] B. Mijvoic, M.D. Vos, I. Gligorijevic, J. Taelman, S.V. Huffel, IEEE Trans. Bio-Med. Eng. 57, 2188 (2010).

[16] P. Common, Sign. Process. 36, 287 (1994).

[17] A. Hyvarinen, IEEE Trans. Neural Networks 10, 626 (1999).

[18] L. Xian, K. He, K.K. Lai, Physica A 454, 11 (2016).

[19] L. Yu, S. Wang, K.K. Lai, F. Wen, Neurocomputing 73, 716 (2010)

[20] F.X. Diebold, R.S. Mariano, J. Busin. Econom. Statist. 20, 134 (1994). 